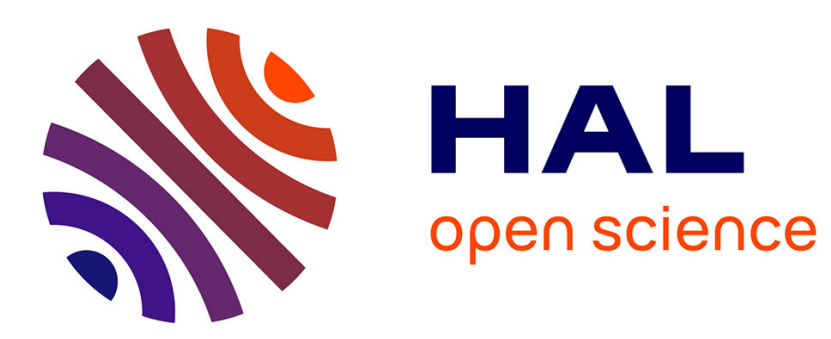

\title{
Characterization of Alfvenic fluctuations in the magnetopause boundary layer
}

\author{
L. Rezeau, A. Morane, S. Perraut, A. Roux, R. Schmidt
}

\section{To cite this version:}

L. Rezeau, A. Morane, S. Perraut, A. Roux, R. Schmidt. Characterization of Alfvenic fluctuations in the magnetopause boundary layer. Journal of Geophysical Research Space Physics, 1989, 94, pp.101110. 10.1029/JA094IA01P00101 . hal-00408546

\section{HAL Id: hal-00408546 https://hal.science/hal-00408546}

Submitted on 8 Jul 2021

HAL is a multi-disciplinary open access archive for the deposit and dissemination of scientific research documents, whether they are published or not. The documents may come from teaching and research institutions in France or abroad, or from public or private research centers.
L'archive ouverte pluridisciplinaire HAL, est destinée au dépôt et à la diffusion de documents scientifiques de niveau recherche, publiés ou non, émanant des établissements d'enseignement et de recherche français ou étrangers, des laboratoires publics ou privés.

$$
\text { Copyright }
$$




\title{
Characterization of Alfvenic Fluctuations in the Magnetopause Boundary Layer
}

\author{
L. Rezeau, A. Morane, S. Perraut, AND A. Roux \\ Centre de Recherches en Physique de l'Environnement, \\ Centre National d'Etudes des Telécommunications, Issy-les-Moulineaux, France \\ R. SCHMDT \\ Space Science Department, European Space Research and Technology Center, Noordwijk, The Netherlands
}

\begin{abstract}
The European Space Agency GEOS 2 spacecraft happened to cross the magnetopause several times, at various local times. Intense electric and magnetic fluctuations, in the ultralow-frequency (ULF) range $(0-10 \mathrm{~Hz})$ have been detected during each such crossing, with a peak at the magnetopause and still large amplitudes in the adjacent magnetosheath and magnetopause boundary layer. By applying spectral analysis and correlations to the electric and magnetic fluctuations, and a minimum variance analysis to the magnetic fluctuations, we investigate the nature of these fluctuations which appear as short-lasting bursts in the spacecraft frame. Having reviewed possible interpretations, we show that the observed electric and magnetic signatures are consistent with small-scale ( $L \approx$ ion Larmor radius) Alfvenic field-aligned structures passing by the spacecraft at high speed. It is suggested that these structures correspond to nonlinear Alfvenic structures.
\end{abstract}

\section{INTRODUCTION}

The geometry of the outer magnetosphere, and the composition, transport, and energization of the plasma within the magnetospheric cavity, are directly related to the rate of transfer of mass and momentum across the magnetopause. Different models which account for these transfers have been considered: the reconnection can be steady and start at a single $X$ line, as proposed originally by Dungey [1961] and developed by many other authors; or it can be nonsteady or patchy, as evidenced by Russell and Elphic [1979]. In either case there is a need for magnetic diffusion (nonideal MHD effects) in a small fraction of the magnetopause or at the locations where the flux transfer events (FTE) develop. On the other hand the diffusion model discussed by Tsurutani and Thorne [1982] is even more demanding because it relies on the assumption that there is magnetic diffusion all over the magnetopause surface.

Therefore, whatever the model of magnetopause, a magnetic diffusion process must be found. As binary collisions are absent in the regions we consider, classical resistivity cannot account for the diffusion, and that has led us to invoke anomalous resistivity associated with high-frequency plasma turbulence; but so far, evidence for waves of the appropriate amplitude at the plasma frequency or at the lower hybrid frequency is scarce. However, the analysis of data from GEOS 2 has led Perraut et al. [1979] and Gendrin [1983] to conclude that a high level of ultralow-frequency fluctuations $(\delta B / B=5 \%)$ is often observed at the magnetopause and that it could account for the required magnetic diffusion. Similar

Copyright 1989 by the American Geophysical Union.

Paper number 8A9512

0148-0227/89/008A-9512\$05.00 magnetic fluctuations have also been detected by the Active Magnetospheric Particle Tracer Explorers (AMPTE) UKS flux gate magnetometer in flux transfer events [Rijnbeek et al., 1987].

A detailed statistical analysis of the spectral properties of magnetic fluctuations observed during magnetopause crossings from GEOS 2 ULF data was recently carried out by Rezeau et al. [1986]. Their study suggests (see Figure 1) that the magnetopause and magnetopause boundary layer (BL) are likely to be the source of the fluctuations observed in the adjacent magnetosheath and magnetosphere. The purpose of this paper is to understand whether the turbulence we observe can be interpreted as plane waves or as signatures of the crossing of nonlinear structures such as Alfven solitons [Hasegawa and Mima, 1976; Sheerin and Ong, 1980]. To complete the characterization of these fluctuations we will (1) make a detailed comparison between electric and magnetic components (by means of correlations and computation of spectra) and (2) study their isotropy, by using a minimum variance analysis.

\section{OBSERVATIONS}

The geostationary orbit of the European Space Agency GEOS 2 spacecraft was not designed to study the structure of the magnetopause because the solar wind ram pressure is seldom large enough to make the magnetopause reach 6.6 Earth radii. However, during 1978 , we were able to find five days (August 27 and 28, September 29, November 12, and December 14) where the spacecraft encountered the magnetopause about 80 times and spent about 12 hours in the magnetosheath, the boundary layer, and the adjacent magnetosphere (it must be noted that during these periods the interplanetary magnetic field was always southward). For these days, data from three magnetic antennas and one electric antenna are available. Electric data, however, suffer from a 
GEOS- 2 AUG . 28,1978 05.00 UT
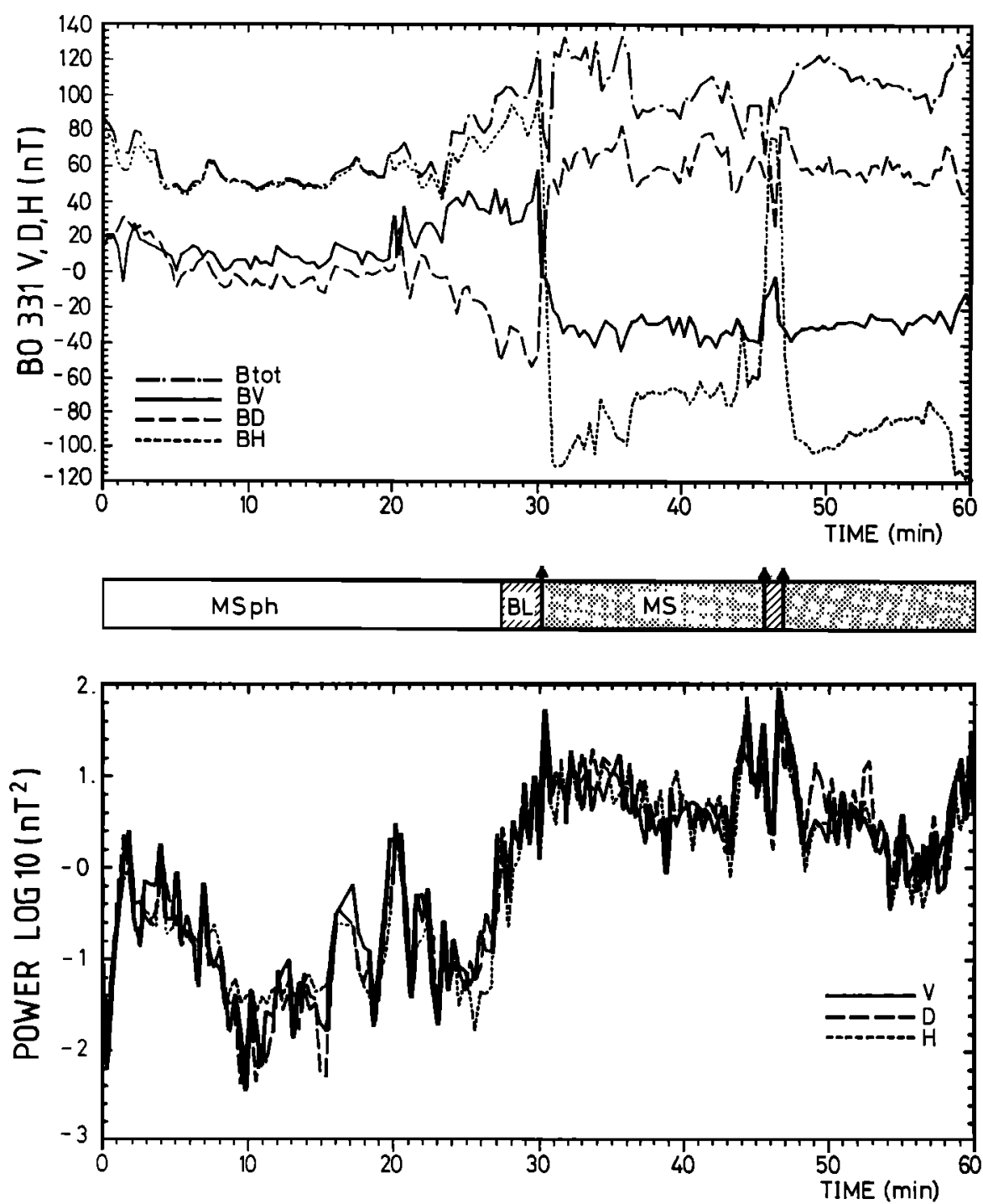

Fig. 1. From Rezeau et al., [1986]; (top) de magnetic field variations over 1 hour (in VDH frame) which shows the localization of the magnetopause (arrows) and of the magnetosphere (MSph), the boundary layer (BL), and the magnetosheath (MS). (Bottom) The power of the fluctuations in the same frame, integrated over $22 \mathrm{~s}$ between 0.5 and 10 Hz.

truncation of about half of each spin period due to a failure in a solar cell panel. In spite of this truncation and of the saturation of the preamplifier sometimes when the signal was too high, we were able to make a systematic study of about 70 crossings (out of 80), located between 0800 and 1300 local time (magnetic latitude $<3^{\circ}$ ). From that study we can get statistically meaningful results, valid at least when the solar wind ram pressure is large enough.

The first step in the analysis is to determine precisely the positions of the different regions (magnetosheath, BL, magnetosphere) along the spacecraft trajectory and the times of crossing of the boundaries between these regions. For that purpose we have used the $H$ component of the dc magnetic field, whose reversal gives the position of the magnetopause (see the upper panel of Figure 1), because owing to the equatorial orbit of GEOS 2, the $V D H$ frame (centered on the spacecraft, with the $V$ direction parallel to the local vertical direction and the $H$ direction horizontal directed toward north) is suitable for studies of the magnetopause (the magnetospheric field is parallel to $H$, and the magnetopause normal is not far from the $V$ direction). We also need the variations of the density which indicate whether we are in the magnetosphere or in the boundary layer. This determination is not easy to achieve. While we have precise and reliable density measurements from the GEOS relaxation sounder [Belmont et al., 1984], this instrument was not designed for continuous operations; it usually gives one point every $10 \mathrm{~min}$, which is inadequate to follow density variations across sharp boundaries such as those studied here. Hence we have used the probe-satellite potential to evaluate density changes. The method is described by Knott et al. [1983]. So far it has only been shown to provide a quantitative estimate of the density in 
GEOS-2, AUG.28, 1978

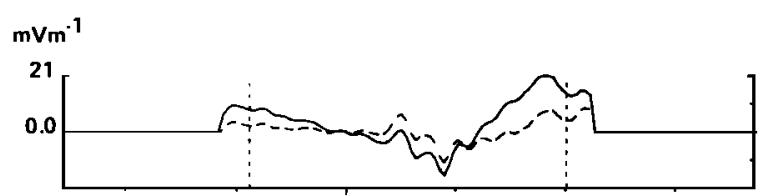

$9: 16: 12$

ELECTRIC (Y) COMPONENT

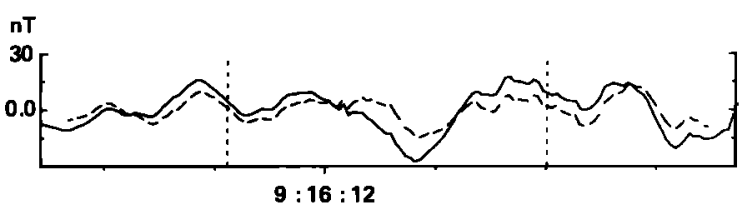

MAGNETIC $(X)$ CONIPONENT

WAVE FORM OF ULTRA - LOW - FREQUENCY WAVES
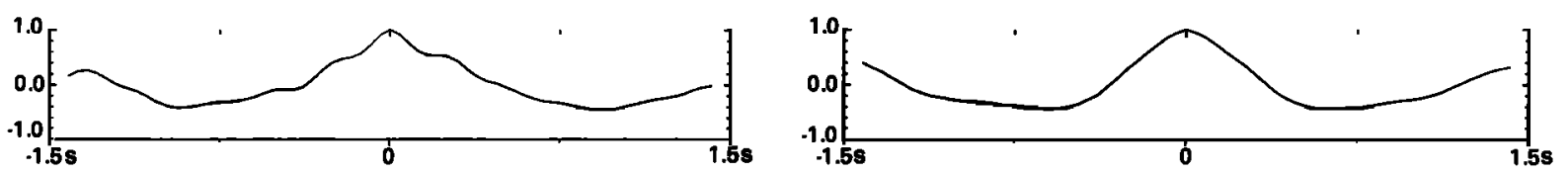

AUTOCORRELATION FUNCTIONS

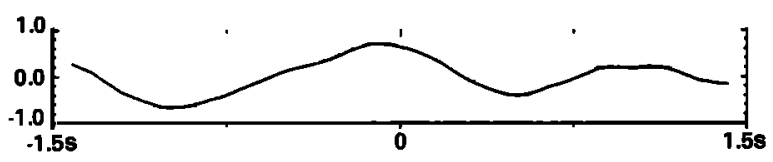

CROSSCORRELATION FUNCTION

Fig. 2. Electric $(Y)$ and magnetic $(X)$ components of the fluctuations in the satellite frame. The dashed lines represent the signal minus a running average calculated over 1 second; the electric data are truncated because of the failure of a solar array, and thus the correlations are calculated over $2.9 \mathrm{~s}$. Beneath the waveforms are the autocorrelation functions and the cross-correlation function.

regions where cold plasma prevails, but it has not been tested in the $\mathrm{BL}$ or the magnetosheath. Therefore we use the probe-satellite potential only as an indicator of the crossing of the boundary between the magnetosphere and the BL. To get approximate values of the plasma density to calculate Alfven velocities in the magnetosheath, we have used the model of Spreiter et al. [1968]. Using the solar wind parameters for a given date and hour [King, 1983], we calculate the Mach number and deduce the average density for each day, from the model. Even though neither method should give exact results, we find that the orders of magnitude of the obtained densities are the same, and that they are quite different from day to day (from $10-20 \mathrm{~cm}^{-3}$ on September 29 to $100-200 \mathrm{~cm}^{-3}$ on August 27).

\section{DATA ANALYSIS}

In this section we (1) investigate the nature of the fluctuations (electromagnetic or electrostatic), (2) investigate their isotropy, and (3) analyze their spectral properties.

\subsection{Electromagnetic Nature of the Fluctuations}

Figure 2 shows an example of the waveforms of the fluctuations (one magnetic and one electric component) on August 28, 1978, at 0916:11 UT, and, beneath, the autocorrelation functions of these components and their cross-correlation function. At this time, GEOS 2 was in the magnetosheath $12 \mathrm{~s}$ before a magnetopause crossing. The data are presented in a satellite frame. Indeed, given that only one electric component is measured, it does not make sense to display the data in a fixed frame. Hence we have to apply a filtering to the data in order to get rid of the spacecraft spin effects (spin frequency $\approx 0.18 \mathrm{~Hz}$ ). We substract a running average (calculated over $1 \mathrm{~s}$ ) from the signal, before calculating the correlations. Another problem with the electric component is the failure of one solar array panel, and that failure prevents us from calculating correlations over more than $2.9 \mathrm{~s}$. The first point to notice is the level of the fluctuations: $\delta E \approx 10 \mathrm{mV} \mathrm{m} \mathrm{m}^{-1}$ and $\delta B \approx 15 \mathrm{nT}$, which is very high, if we compare it with what is measured in the magnetosphere in the same frequency range [see Young et al., 1981, Perraut et al., 1982]. The second point is the value of the cross-correlation function, $\approx 0.7 ;$ it is also worth noticing that the autocorrelation functions obtained for both components are very similar. This good correlation between electric and magnetic components clearly shows that we are observing electromagnetic fluctuations. From the amplitudes of the components, we can estimate $\delta E / \delta B \approx 670$ $\mathrm{km} \mathrm{s}^{-1}$. If we assume that this is the Alfven velocity we must compare it to $V_{A}=\left(B_{0} 2 / \mu_{0} n m_{\mathrm{i}}\right)^{0.5}$, where $B_{0}=100 \mathrm{nT}$ is the average value of the magnetic field at that time and where $n$ is not known precisely. The value of the density corresponding to $V_{A}=670 \mathrm{~km} \mathrm{~s}^{-1}$ is $n \approx 11 \mathrm{~cm}^{-3}$, which 

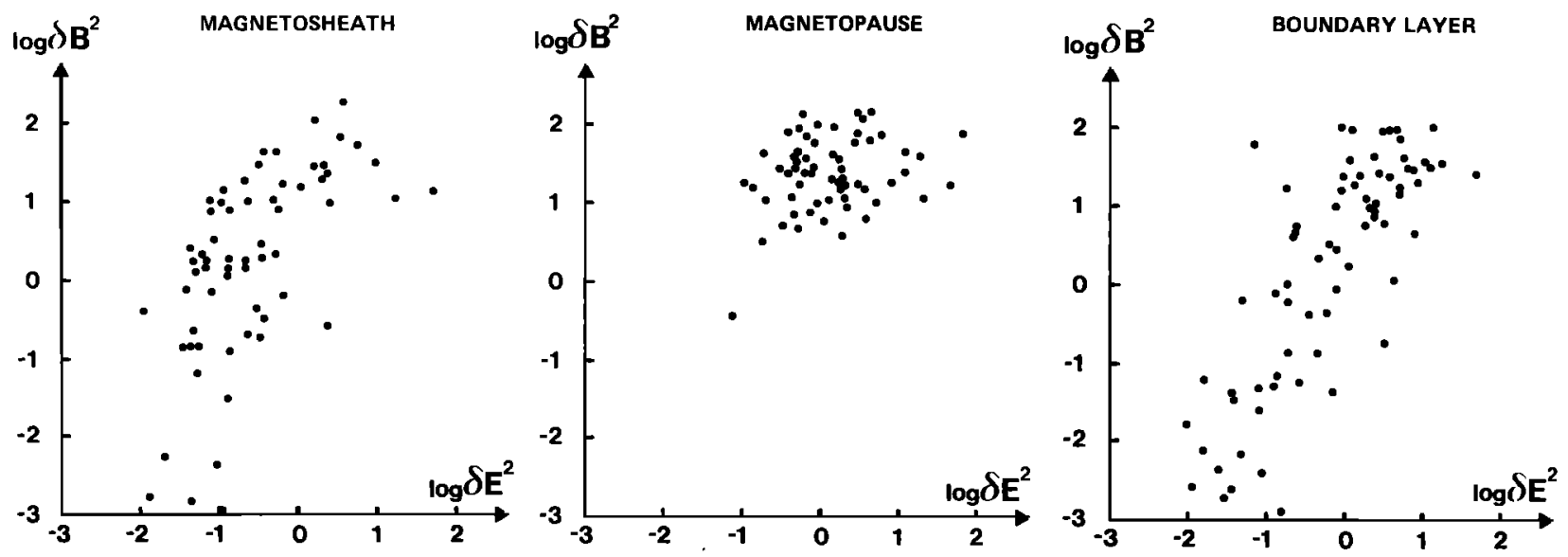

Fig. 3. Comparison between electric and magnetic powers integrated between 1 and $11 \mathrm{~Hz}$, over $22 \mathrm{~s}$, in logarithmic scale (the electric power is calculated from the only available component). Each point represents a time interval of $22 \mathrm{~s}$.

is 2-3 times too low compared to what we might expect. But given that we do not measure all the components of the electric field and that we can only guess the order of magnitude of the density, it still indicates that the fluctuations are Alfvenic, but does not allow us to fully assess their nature. For that we have to consider the other components. In the present case, cross-correlations with the other components give about the same results. We shall further study the isotropy of the magnetic fluctuations in the next section.

In order to generalize the above result we compute the power of the electric component and the sum of the powers of the magnetic components, both integrated over $22 \mathrm{~s}$ between 1 and $11 \mathrm{~Hz}$, for a large number of cases. At the magnetopause we select each period of time centered on a crossing; in the other regions we select, at random, periods of $22 \mathrm{~s}$ so as to obtain about the same number of cases in each region (around 70). The magnetic power versus the electric power is plotted in Figure 3 , in $\log -\log$ scale, for the magnetosheath, magnetopause, and $B L$, respectively. For the magnetosheath and $B L$, data can be fitted by straight lines. Since the slopes of these lines are of the order of $1, \delta E^{2} \propto \delta B^{2}$, and we can check whether the factor of proportionality is of the order of an Alfven velocity, as it should be for Alfvenic fluctuations (it must be noticed that we have used only one electric component and therefore we can only underestimate $V_{A}$ ). We find $V_{A}=380 \mathrm{~km} \mathrm{~s}^{-1}$ in the magnetosheath and $600 \mathrm{~km} \mathrm{~s}^{-1}$ in the $B L$. If we assume $B_{0} \approx 100 \mathrm{nT}$ (which is the average value of the field in the regions we study), these values correspond to densities of $30 \mathrm{~cm}^{-3}$ and $13 \mathrm{~cm}^{-3}$, respectively, which basically agree with the expected orders of magnitude of densities in these regions (averaged over all days). We conclude that the fluctuations we observe on electric and magnetic antennas are Alfvenic, at least in the magnetosheath and the BL. For the magnetopause itself it is more risky to draw the same conclusion because the range of observed amplitudes is too small to enable us to make any reasonable fit.

\subsection{Voriance Analysis}

The results presented in the previous section suggest that we observe waves. In order to check this, it is useful to study the polarization of the fluctuations. Because we only have one electric component, we have used here the three magnetic components and applied to them a minimum variance analysis, as described by Belcher and Davis [1971]. We compute a variance matrix over $3 \mathrm{~s}$ of the signal filtered at $0.5 \mathrm{~Hz}$ to get rid of spin effects. After diagonalization, we obtain three eigenvalues $\left(\sigma_{i}\right)$ and three eigenvectors $\left(u_{i}\right), i=1,2,3$. From the eigenvalues we can get an indication of the isotropy by calculating the ratio $\sigma_{3} / \sigma_{1}$ which should be equal to 1 in the case of a perfect isotropy and much smaller than 1 if the fluctuations were the signatures of plane waves. If the fluctuations are anisotropic, let $u_{3}$ be the direction of minimum variation and $\mathbf{u}_{1}$ the direction of maximum variation.

The anisotropy ratios $\sigma_{3} / \sigma_{1}$ are 0.15 in the boundary layer, 0.16 in the magnetosheath, and 0.22 at the magnetopause: they are intermediate between the two possibilities quoted above. Thus the conclusion is that the fluctuations are not plane waves, but they do not correspond either to the isotropic turbulence usually studied in turbulence theories.

Let us now investigate the directions of anisotropy of the fluctuations. The histograms of the distributions of angles between the $u_{i}$ directions and $B_{0}$ are plotted in Figure 4. They show an average direction of maximum variation which is clearly perpendicular to the dc magnetic field $B_{0}$. The direction of minimum variation is less clearly determined; still it tends to be parallel to the dc magnetic field. For the time intervals chosen corresponding to the magnetopause, the results are plotted in the $V D H$ frame because a frame with the $Z$ axis parallel to the dc magnetic field does not make any sense at the magnetopause. The $H$ direction roughly indicates the direction of the magnetic field in the neighboring magnetosphere. 

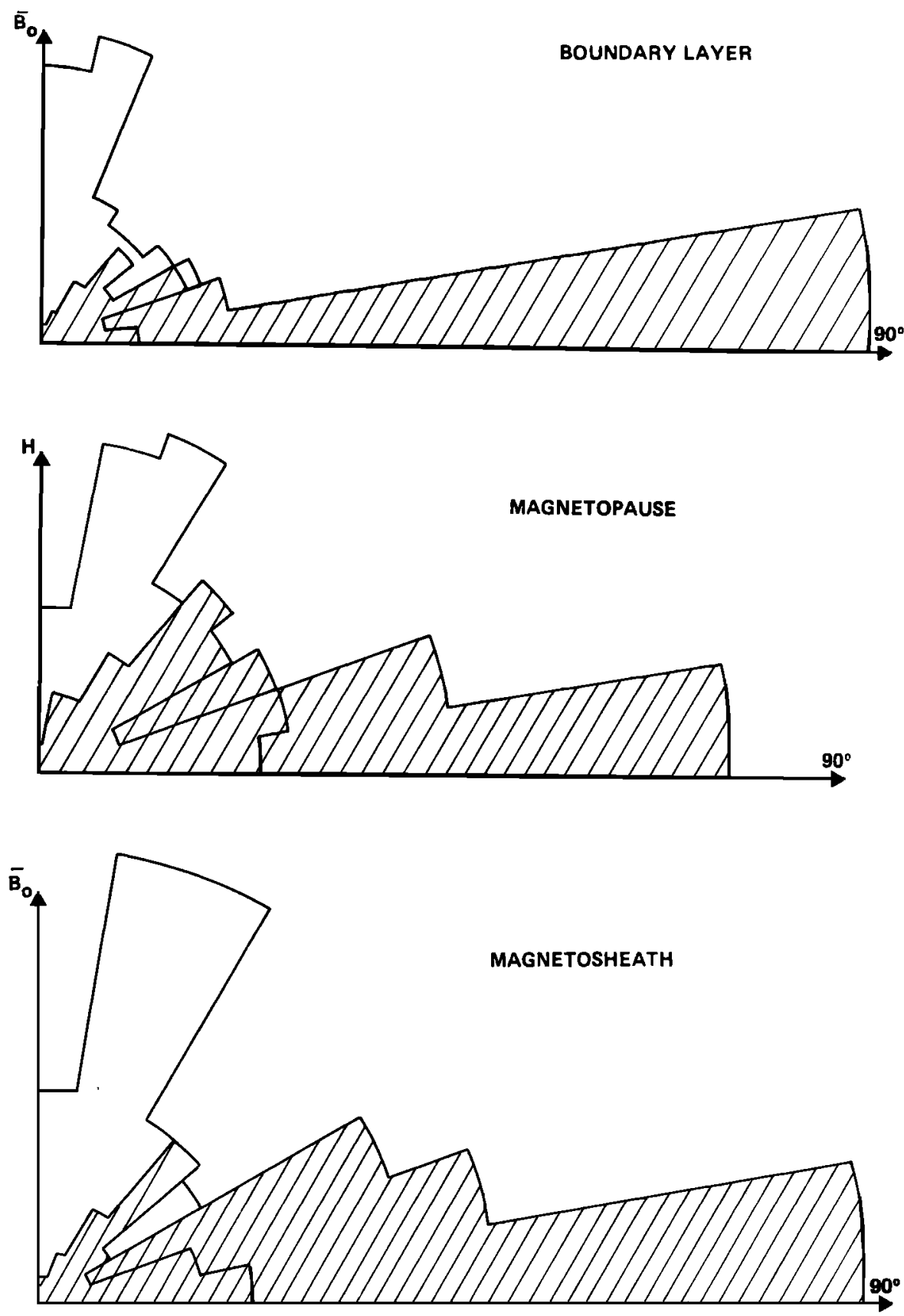

Fig. 4. Histograms of the distributions of angle between the minimum variance direction and a reference direction (nonhatched distribution), and of the distribution of angle between the maximum variance direction and the same reference direction (hatched distribution). The reference direction is the dc magnetic field $B_{0}$ in the boundary layer and the magnetosheath, the $\boldsymbol{H}$ direction at the magnetopause.

\subsection{Spectral Analysis of the Fluctuations}

Frequency spectra of the electric and magnetic components are displayed in Figure 5 for one magnetopause crossing. No clear peak shows up; instead the spectral density is, on average, a monotonous decreasing function of frequency. This was already pointed out by Rezeau et al. [1986], who showed that these spectra can be fitted by a $p_{0} f^{-\alpha}$ law. The results presented in this subsection complete those of Rezeau et al. and extend the study to the electric component. Because of the truncation of the data (see Figure 2), the electric spectra are computed over $2.9 \mathrm{~s}$ during each spin period; for the magnetic components, as there is no truncation of the data, we have computed the spectra over $5.5 \mathrm{~s}$ (approximately the spin period). Although for the electric field we have to work in the satellite frame, it is more appropriate for investigating the possible anisotropy of the indices to make the fits of the magnetic spectra in a frame with the $Z$ axis along the dc magnetic field. Then four successive spectra are averaged to obtain a total duration of $22 \mathrm{~s}$. The fit is made through a linear 


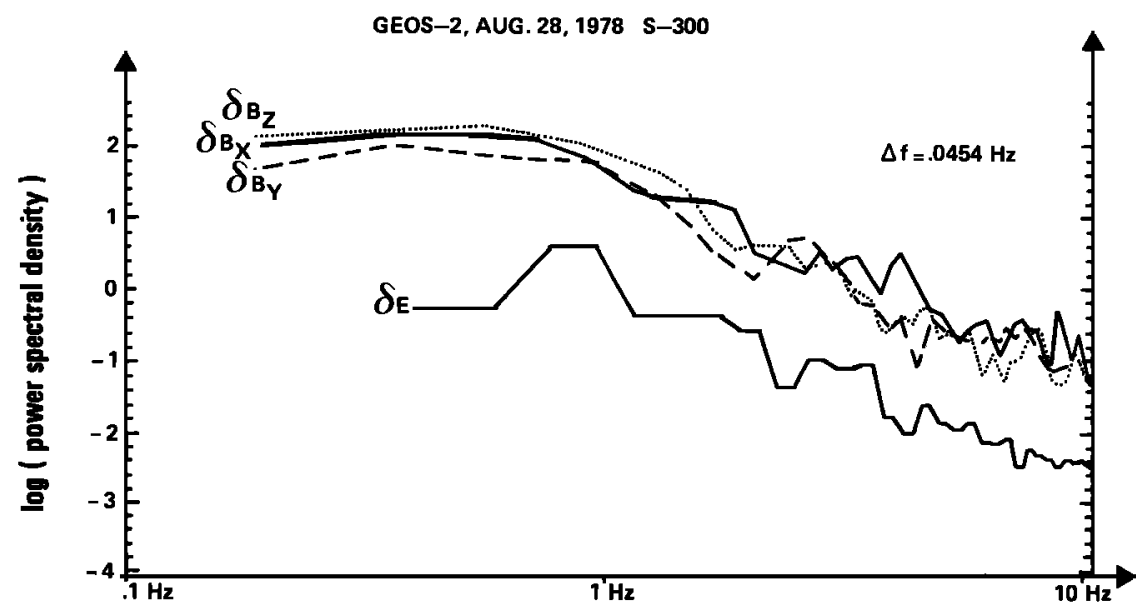

\begin{tabular}{|l|l|l|l|l|}
\hline & $\log \rho_{\mathrm{o}}$ & $\alpha$ & $\epsilon_{\mathrm{o}}$ & $\epsilon_{1}$ \\
\hline $\mathrm{X}$ & 1.56 & 2.56 & .098 & .128 \\
$\mathrm{Y}$ & 1.26 & 2.44 & .093 & .122 \\
$\mathrm{Z}$ & 1.44 & 2.66 & .108 & .142 \\
$\mathrm{E}$ & 0.07 & 2.58 & .073 & .100 \\
\hline
\end{tabular}

Fig. 5. Example of magnetic and electric spectra, computed over 5.5 and $2.9 \mathrm{~s}$, respectively, and averaged over $22 \mathrm{~s}$, at 0731:12 UT on August 28 (magnetopause crossing). The scales for the power spectral densities are in $\mathrm{mV}^{2} \mathrm{~m}^{-2} \mathrm{~Hz}^{-1}$ for the electric component and $\mathrm{nT}^{2} \mathrm{~Hz}^{-1}$ for the magnetic components. The parameters of the linear regression are on the lower panel ( $\varepsilon_{0}$ and $\varepsilon_{\alpha}$ are the errors on the determination of $\log _{0}$ and $\alpha$ ).

regression between 1 and $11 \mathrm{~Hz}$, from which we determine the index $\alpha$, the logarithm of the power spectral density at $1 \mathrm{~Hz}$, $P_{0}$, and the errors on both parameters from a standard statistical method [Mendenhall, 1967]. For the particular spectra of Figure 5 the results are indicated in the lower part of the figure. Indices $\alpha$ were computed for about 80 cases in each region, and the results are shown in Figure 6.

For the magnetic components the conclusions are about the same as those of Rezeau et al. [1986], who used much longer time intervals $(3 \mathrm{~min})$. The shorter time interval used here (22 $s$ instead of $3 \mathrm{~min}$ ) is a better compromise between the need for a good time resolution (because the medium changes fast) and the error on the determination of the index, which is not much larger for $22 \mathrm{~s}$. The region where the fit is the best is the magnetopause; the average of the errors on $\alpha$ is 0.14 , and the statistical error in the determination of $\alpha$ is only 0.09 . The average indices are $2.4,2.6$, and 2.5 for the three components. Within the other regions the fit is less good (the errors on the regression parameters are larger), and the obtained indices are more scattered (statistical error of the order of 0.15 ) than at the magnetopause and always higher than 2.4. (In the magnetosphere, the errors are still larger, but the power of the fluctuations is much lower and the results are not really significant.)

For the electric spectra the fit with a $p_{0} f^{-\alpha}$ law is also generally good, especially in the magnetosheath, where the index $\alpha_{E}$ is the same as for the magnetic components, $\alpha_{X, Y, Z}$. Elsewhere there is a significant difference between the electric and magnetic indices; this difference is quite large at the magnetopause, where the scattering of $\alpha_{E}$ is also larger. An examination of individual spectra of the electric

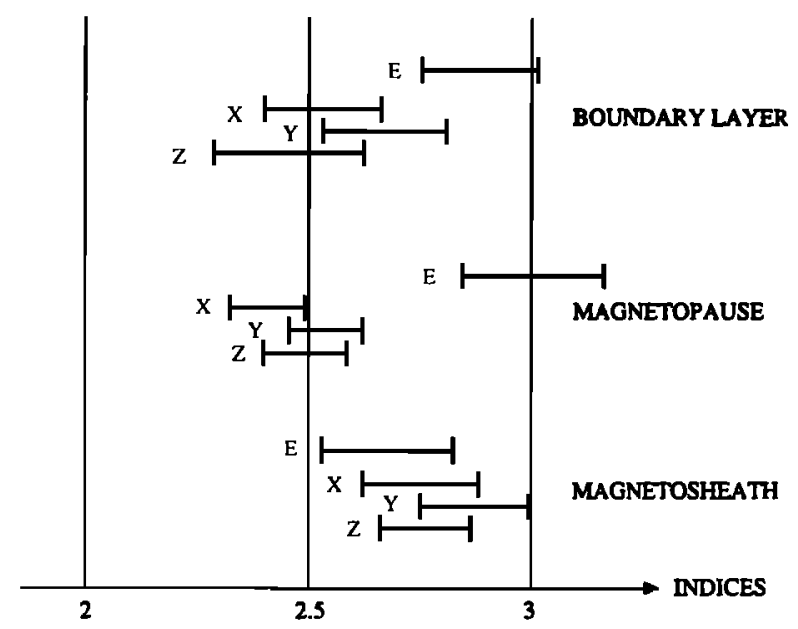

Fig. 6. Statistical results on the indices, depending on the region. The horizontal lines indicate the $95 \%$ confidence intervals in the statistical determination of the average index $\alpha$ for each component. 


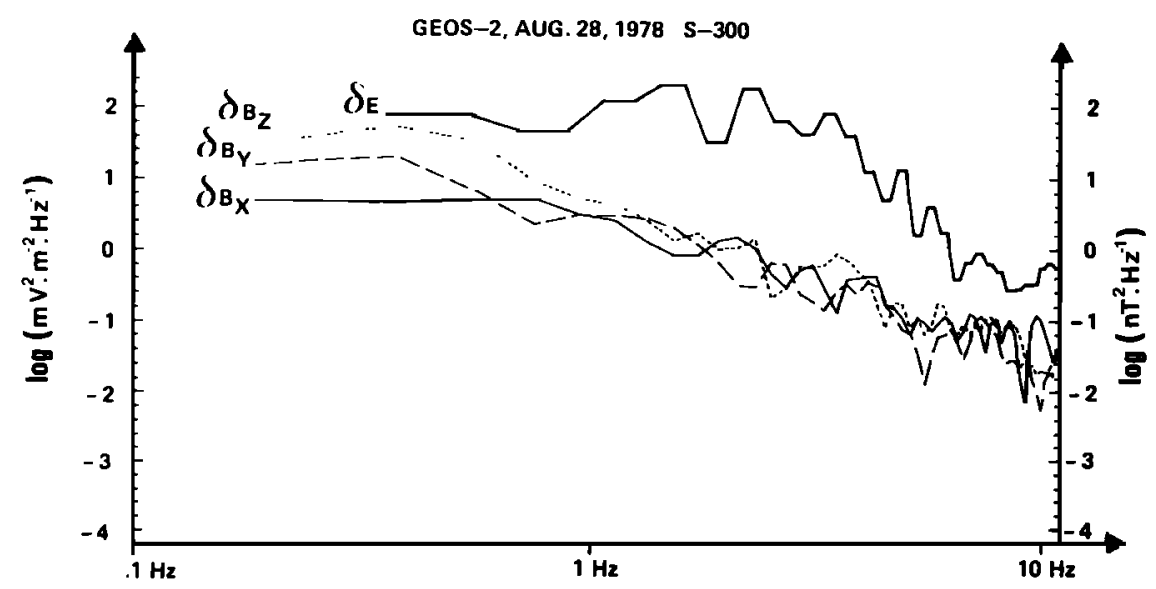

Fig. 7 . Magnetopause crossing spectra, at 0607:15 UT on August 28. The computation is the same as for Figure 5. This is an example where spectral laws for the electric and magnetic components are different (see text).

fluctuations during magnetopause crossings shows that they do not always appear to be smoothly decreasing like the magnetic ones (e.g., Figure 5). Most of them are similar to the example plotted in Figure 7, where the index obtained for the $\delta E$ spectrum is 3.95 with an error of 0.18 . Given the shape of this spectrum, it is not surprising that the fit is not good. That explains why the average index for the magnetopause crossings is so large and brings some doubt about the validity of the fit there.

\section{DISCUSSION AND INTERPRETATION}

Because the magnetic indices are isotropic and their values in each of the regions studied (magnetopause and adjacent magnetosheath and $\mathrm{BL}$ ) are not much scattered, it is tempting to interpret the observed fluctuations as isotropic MHD turbulence [Kraichnan, 1965; Montgomery, 1985]. However, the assumptions made in such theories, namely homogeneity and isotropy of the medium and isotropy of the spectral density, are far from being granted in the regions we investigate. Furthermore, the electric component of the fluctuations shows a different behavior especially at the magnetopause and in the BL. In conclusion it seems that isotropic MHD turbulence theory is not applicable to the present data.

Since the fluctuations are electromagnetic with ratios $\delta E / \delta B$ of the order of the Alfven velocity (except maybe at the magnetopause itself) and the direction of minimum variance is along $B_{0}$, it is tempting to interpret the fluctuations as being plane Alfven waves propagating along $\mathbf{B}_{0}$. This interpretation is not fully adequate, however, because the fluctuations are very intense $\left(\delta E \approx 10 \mathrm{mV} \mathrm{m}^{-1}\right.$ and $\delta B \approx 15 \mathrm{nT}$ ), which suggests that strong nonlinear effects are operative; this is consistent with the fact that the fluctuations are limited in time. This last point can be interpreted in the following way: the magnetosheath plasma, drifting along the magnetopause surface, carries along this surface structures which are confined in a direction perpendicular to $\mathbf{B}_{0}$ (of course this cannot be proved unambiguously with single-satellite measurements). Both points are inconsistent with a plane wave interpretation. Furthermore, the smooth spectra observed at the magnetopause and in the adjacent regions are very different from those of ion cyclotron waves [Young et al., 1981] or magnetosonic waves [Perraut et al., 1982] observed in the inner magnetosphere with the same instrument, in the same frequency range, which exhibit well-defined peaks close to the helium or proton gyrofrequencies.

The analysis of similar (but somewhat weaker) events observed in the night sector has been performed by Robert et al. [1984]. These authors showed that isolated events can be interpreted, if plotted in the right frame, as the signatures of field-aligned current structures passing by the spacecraft at high speed. A search for such signatures has been made on the dayside, during periods when the magnetopause was crossed. A few isolated events were found. One example is shown in Figure $8 a$, where the fluctuations are plotted in the frame obtained by minimum variance analysis. It can be noticed that the minimum variance direction $\left(u_{3}\right)$ is parallel to the dc magnetic field $\left(\theta_{3}=15^{\circ}\right)$ and that the shape of the two other components is as expected for a field-aligned current structure passing by the spacecraft. The shape of the current is in that case more elliptical than cylindrical, as can be seen on the hodogram drawn in the $u_{1}, u_{2}$ plane (Figure $8 b$ ). The model of Robert et al. [1984] does not account for the simultaneous observation of electric field fluctuations, correlated with magnetic ones (see Figure 2). Hence we are led to conclude that we are observing nonstationary spatially limited structures. Furthermore, the scarcity of isolated events suggests that there are several such structures passing close to the spacecraft, thus leading to the complex waveform which is usually observed.

The existence of such nonlinear structures has already been investigated in a number of theoretical works. Hasegawa and Mima [1976] and Chmyrev and Mordovskaia [1985] studied the problem in plane geometry, and Sheerin and Ong [1980] used a cylindrical geometry which seems more appropriate to describe our results. It is interesting to notice that the typical transverse scale found by Sheerin and Ong [1980], the ion 

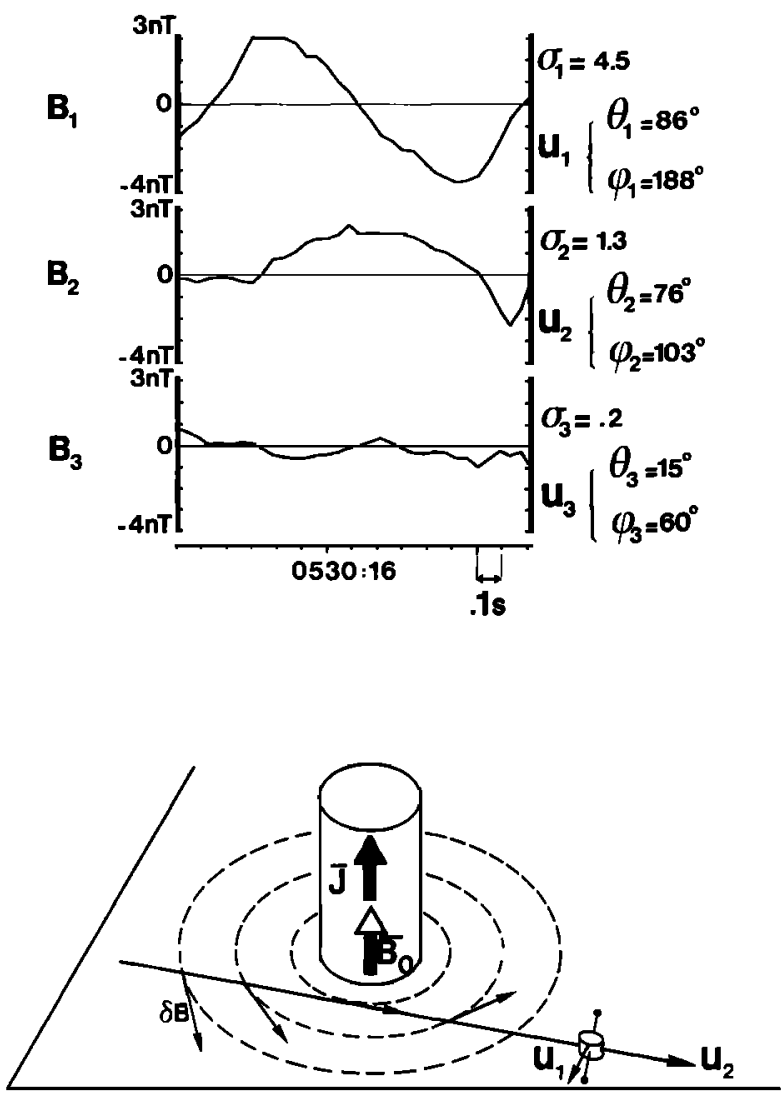

Fig. 8a. Fluctuations in the $\left(u_{1}, u_{2}, u_{3}\right)$ frame, computed over $1.5 \mathrm{~s}$ from a minimum variance analysis. The frame parameters are indicated on the right of the upper panel: variances and vector directions with respect to the dc magnetic field ( $\theta$ angle with the field and $\varphi$ angle in the perpendicular plane).

Larmor radius, fits well with our data if a relative speed of 50 or $100 \mathrm{~km} \mathrm{~s}^{-1}$ is used; that is, if we assume the structures to be moving at the magnetosheath flow velocity, or a fraction of this velocity. However, it is difficult to make a detailed comparison between our data and the results of Sheerin and Ong because (1) they are not analytical and (2) their analysis is restricted to the case $\beta$ « 1 , which certainly does not apply here. Hence awaiting for a model where $\beta \approx 1$ and cylindrical effects are included, we have tried to fit the data with a simpler analytical model. The basic assumption made is that a nonlinear structure, limited in space, passes by the satellite. However, we assume that the structure itself is not crossed and hence nonlinear effects which are likely to take place within the structure may be neglected. Then we only consider the electromagnetic field which is radiated out of the structure. In the magnetopause itself where the intensity is very large it is likely that the structures themselves are often crossed, which certainly makes the above assumption very poor. In the adjacent regions, however, we are certainly more often close to nonlinear structures than within them. Hence the above approximation, based on a linear model in cylindrical geometry, is probably a good guide for analyzing the data in the regions adjacent to the magnetopause, more than in the magnetopause itself.
We write the fundamental system of equations for electromagnetic fields and plasma quantities: Maxwell-Faraday and Maxwell-Ampère laws and conservation of mass and momentum. We make the usual assumption of quasi-neutrality and neglect electron inertia. As we consider a six-component electromagnetic field, the resolution of the system is not straightforward, and by now we have solved it only in the case of a cold plasma, which is not really satisfactory in the regions studied here. We also assume an axial symmetry (no azimuthal variation). Following Sheerin and Ong [1980], we choose a coordinate parallel to the magnetic field $\xi=z-u t$. Finally we assume parallel propagation in the form $\exp (i x \xi)$. thus reducing the variables to only one, the radial direction $r$. Making use of these hypothesis, we find the following system ( $\rho_{0}$ is the equilibrium density):

$$
\begin{aligned}
& -i u \kappa \rho_{0} v_{r}=\frac{B_{0}}{\mu_{0}}\left(i \kappa B_{r}-\frac{d B_{z}}{d}\right) \\
& -i u \kappa \rho_{0} v_{\theta}=i \kappa \frac{B_{0}}{\mu_{0}} B_{\theta} \\
& -i u \kappa \rho_{0} v_{2}=0 \\
& -i \kappa\left(v_{r} B_{0}-i \frac{m_{i}}{e} u \kappa v_{\theta}\right)=i u \kappa B_{r} \\
& i \kappa\left(-v_{\theta} B_{0}-i \frac{m_{i}}{e} u \kappa v_{r}\right)+i \frac{m_{i}}{e} u \kappa \frac{d v_{z}}{d}=i u \kappa B_{\theta} \\
& \frac{1 d}{r d}\left[r\left(v_{r} B_{0}-\frac{m_{i}}{e} u \kappa v_{\theta}\right)\right]=i u \kappa B_{z}
\end{aligned}
$$

The solution of this system gives the following equation for the azimuthal component of the magnetic field $B_{\theta}\left(V_{A}\right.$ is the Alfven speed and $\omega_{i}$ the ion gyrofrequency):

$$
\frac{d}{d}\left(\frac{1}{r} \frac{d}{d r}\left(r B_{\theta}\right)\right)+C B_{\theta}=0
$$

The $C$ constant is defined by the following formula:

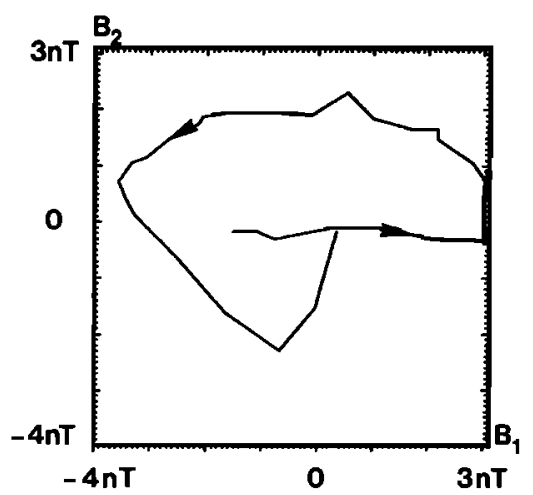

Fig. $8 b$. Hodogram of the fluctuations in the $u_{1}, u_{2}$ plane. The arrows indicate the direction of rotation of $\mathbf{\delta B}$. 


$$
C=-\kappa^{2}\left(\begin{array}{c}
1-\frac{u^{2}}{v_{A}^{2}} \\
1+\frac{v_{A}^{2}}{u^{2}}+\frac{\kappa^{2} v_{A}^{2}}{\omega_{i}^{2}}
\end{array}\right)
$$

The solution of equation (1) is a Bessel function $J_{1}$ or $K_{1}$, depending on the sign of $C$, given by equation (2). The case which seems the most interesting in order to interpret the data is the case of an oscillating function $J_{1}$, which is possible to obtain when $u<V_{A}$. The parallel phase velocity $u$ is equal to $V_{A}$ for an ideal MHD shear Alfven wave. If dispersion terms are taken into account (in the present case ion inertia effects), the dispersion relation becomes $\omega^{2}=k / I^{2} V_{A}{ }^{2}\left(1-\left(k^{2} / k_{\perp}{ }^{2}\right)\left(\omega^{2} / \omega_{i}{ }^{2}\right)\right)$, which implies $u<V_{A}$. On the other hand the case $u>V_{A}$ corresponds to the compressional mode.

Then, further assuming that the structure is moving with respect to the spacecraft in a direction perpendicular to the magnetic field, we find $B_{\theta}$ as a function of time through the argument $\left(C\left(V^{2} t^{2}+a^{2}\right)\right)^{0.5}$, where $a$ is the impact parameter and $V$ the relative speed. To compare with the experimental spectral laws, we calculate the Fourier transform of this function over a given time interval. The order of magnitude of the drift velocity of the magnetosheath flow (which carries along the structures) is $100 \mathrm{~km} \mathrm{~s}^{-1}$. As we do not know the parameters of the wave $(a, C)$, we adjust them in order to satisfy two conditions: (1) the center of the structure must not be crossed (which means that $C a^{2}$ is taken large enough so that the first maximum of $J_{1}$ is not reached), and (2) the variation of the argument must be large enough to explore several oscillations. We then obtain a spectral law which can be fitted by $f^{-3.3}$. This is not exactly the spectral index we found experimentally, but given the simplicity of the model we feel the agreement is quite good. At least the comparison between this simple model and the data suggests that the smoothly decreasing spectra observed especially in the magnetosheath and the BL are essentially due to moving electromagnetic structures passing by the spacecraft at a high speed. This is just what we expect for the nonlinear version of kinetic Alfven waves which discharge the reconnecting magnetopause onto the upper resistive ionosphere. We hope this work, which gives experimental evidence for nonlinear Alfven waves in a high $\beta$ plasma $(\beta \approx 1)$, will stimulate further theoretical efforts.

Note added in proof. In the meantime we have extended the above model, including thermal effects. The algebra is more cumbersome, but the main result is the same: the radial dependence of the magnetic perturbation is a $J_{1}$ or a $K_{1}$ function.

Acknowledgements. The Editor thanks two referees for their assistance in evaluating this paper.

\section{REFERENCES}

Belcher, J. W., and L. Davis, Jr., Large amplitude Alfven waves in the interplanetary medium, 2, J. Geophys. Res., 76, 3534-3563, 1971.

Belmont, G., P. Canu, J. Etcheto, H. de Feraudy, and B. Higel, Contribution of relaxation sounding experiments to the understanding of magnetospheric processes, in Proceedings of Conference on Achievements of the IMS, June 1984, Eur. Space Agency Spec. Publ., ESA/SP-217, 701-704, 1984.

Chmyrev, V. M., and V. G. Mordovskaia, Non-linear theory of electromagnetic bursts in plasma near the Earth, Preprint 534, ZZMIRAN, Moscow, 1985.

Dungey, J. W., Interplanetary magnetic field and the auroral zones, Phys. Rev. Lett., 6, 47-48, 1961.

Gendrin, R., Magnetic turbulence and diffusion processes in the magnetopause boundary layer, Geophys. Res. Lett., 10, 769-771, 1983.

Hasegawa, A., and K. Mima, Exact solitary Alfven wave, Phys. Rev. Lett., 37, 690-693, 1976.

King, J. H., Interplanetary medium data book, supplement 2, Rep. NSSDC/WDC-A-R\&S, Natl. Space Sci. Data Cent., Greenbelt, Md., 1983.

Knott, K., P. Decreau, A. Korth, A. Pedersen, and G. Wrenn, Observations of the GEOS equilibrium potential and its relation to ambient electron energy distribution, in Proceedings of the 17th ESLAB Symposium on Spacecraft: Plasma Interactions and Their Influence on Field and Particle Measurements, Noordwijk, Sept. 1983, Eur. Space Agency Spec. Publ., ESA/SP.198, 19-24, 1983.

Kraichnan, R. H., Initial-range spectrum of hydromagnetic turbulence, Phys. Fluids, 8, 1385-1387, 1965.

Mendenhall, W., Introduction to Probability and Statistics, Wadsworth, Belmont, Calif., 1967.

Montgomery, D., Characteristic phenomena of magnetohydrodynamic turbulence, Eur. Space Agency Spec. Publ., ESA/SP-235, 175-179, 1985.

Perraut, S., R. Gendrin, P. Robert, and A. Roux, Magnetic pulsations observed onboard GEOS 2 in the ULF range during multiple magnetopause crossings, in Proceedings of Magnetospheric Boundary Layer Conference, Alpach, June 1979, Eur. Space Agency Spec. Publ., ESA/SP-148, 113-122, 1979.

Perraut, S., A. Roux, P. Robert, R. Gendrin, J. A. Sauvaud, J. M. Bosqued, G. Kremser, and A. Korth, A systematic study of ULF waves above $F_{\mathrm{H}}$ from GEOS 1 and 2 measurements and their relationship with proton ring distributions, J. Geophys. Res., 87, 6219-6236, 1982.

Rezeau, L., S. Perraut, and A. Roux, Electromagnetic fluctuations in the vicinity of the magnetopause, Geophys. Res. Lett., 13, 1093-1096, 1986.

Rijnbeek, R. P., C. J. Farnugia, D. J. Southwood, M. W. Dunlop, W. A. C. Mier Jedrzejowicz, C. P. Chaloner, D. S. Hall, and M. F. Smith, A magnetic boundary signature within flux transfer events, Planet. Space Sci., 35, 871-878, 1987.

Robert, P., R. Gendrin, S. Perraut, and A. Roux, GEOS 2 identification of rapidly moving current structures in the equatorial outer magnetosphere during substorms, J. Geophys. Res., 89, 819-840, 1984.

Russell, C. T., and R. C. Elphic, ISEE observations of flux transfer events at the dayside magnetopause, Geophys. Res. Lett., 6, 33-36, 1979.

Sheerin, J. P., and R. S. B. Ong, Solitary Alfven waveguide structures in a magnetized plasma, J. Plasma Phys., 24, 157-162, 1980.

Spreiter, J., A. Alksne, and A. Summers, Extemal aerodynamics of the magnetosphere, in Physics of the Magnetosphere, edited by R.L. Carovillano, D. Reidel, Dordrecht, Holland. 1968. 
Tsurutani, B. T., and R. M. Thome, Diffusion processes in the magnetopause boundary layer, Geophys. Res. Lett., 9, 1247-1250, 1982.

Young, D. T., S. Perraut, C. de Villedary, R. Gendrin, A. Korth, G. Kremser, and D. Jones, Wave-particle interactions near $\Omega_{\mathrm{He}}+$ observed on GEOS 1 and GEOS 2, 1, Propagation of ion cyclotron waves in $\mathrm{He}^{+}$rich plasma, J. Geophys. Res., 86, 6755-6772, 1981.
A. Morane, S. Perraut, L. Rezeau, and A. Roux, CRPE/CNET, 92131 Issy-les-Moulineaux Cédex, France.

R. Schmidt, SSD/ESTEC, 2200 AG-Noordwijk, The Netherlands.

(Received January 20, 1988;

revised March 24, 1988;

accepted April 13, 1988.) 